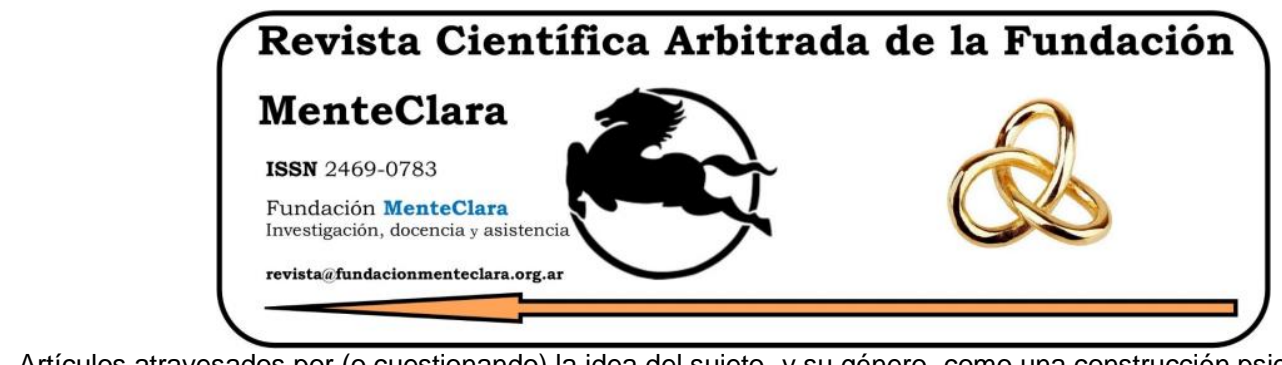

Artículos atravesados por (o cuestionando) la idea del sujeto -y su género- como una construcción psicobiológica de la cultura. Articles driven by (or questioning) the idea of the subject -and their gender- as a cultural psychobiological construction.

Vol. 6 (2021), enero-diciembre ISSN 2469-0783

https://datahub.io/dataset/2021-6-e218

\title{
REPRESENTACIONES SOCIALES EN ORGANIZACIONES DE SEGURIDAD. ROL E INFLUENCIA EN EL SENTIDO DE VIDA
}

\author{
SOCIAL REPRESENTATIONS IN SECURITY ORGANIZATIONS. ROLE AND \\ INFLUENCE IN THE MEANING OF LIFE
}

Carolina Viviana Rivela ${ }^{1}$, Analía Verónica Losada²

Cómo citar este artículo / Citation: Rivela, C. V. \& Losada, A. V. (2021).

Representaciones Sociales en Organizaciones de Seguridad. Rol e Influencia en el Sentido de Vida. Revista Científica Arbitrada de la Fundación MenteClara, Vol. 6 (218). DOI: https://doi.org/10.32351/rca.v6.218

Copyright: (C) 2021 RCAFMC. Este artículo de acceso abierto es distribuido bajo los términos de la licencia Creative Commons Attribution 4.0 International License (CC BY 4.0). Recibido: 08/02/2021. Aceptado: 21/02/2021 Publicación online: 22/02/2021

Conflicto de intereses: Ninguno que declarar.

\section{Resumen}

La investigación indagó las representaciones sociales y aspiraciones personales de personas que forman parte de organizaciones de seguridad y su repercusión en el sentido de vida, empleando una orientación metodológica mixta con una muestra de 93 miembros de fuerzas de seguridad. El tipo de diseño utilizado fue no experimental de alcance fenomenológico descriptivo, administrando en el marco de entrevistas los instrumentos de Cuestionario de Apreciación sobre Rol y Aspiraciones Personales (CARAP) y la Adaptación Argentina de Andrés Gottfried del Purpose in Life Test (PIL TEST) de Crumbaugh y Maholick a fin de conocer la medida del sentido de vida y las representaciones sociales. Los hallazgos demuestran que el $80 \%$ de los entrevistados, manifestaron tener su propia idea de rol: el 17,20\% desconocian las tareas y

\footnotetext{
${ }^{1}$ Universidad de Flores, Argentina. carolina.rivela@uflouniversidad.edu.ar

2 Universidad de Flores, Argentina. analia.losada@uflo.edu.ar
} 
actividades relacionadas con el rol a desempeñar y el 2, 15\% restante dio respuestas que no respondian a la pregunta realizada.

Los resultados que corresponden al cuestionario CARAP y parte B del PIL Test permiten afirmar que fue posible conocer las aspiraciones de los participantes que forman parte de organizaciones de seguridad. Asimismo, se evidenció que para lograr sus aspiraciones en un porcentaje significativo del 46,49\% reconocen la necesidad de esforzarse, dar de sí mismo y tener que trabajar para alcanzar lo que anhelan. Por último, se detalló una propuesta para enriquecer los procesos de formación de futuros aspirantes al recibir orientación con dinámicas grupales e iniciarse en la reflexión sobre el rol que desempeñaran y la influencia que tendrá en su sentido de vida.

\section{Abstract}

The research investigated the social representations and personal aspirations of people who take part in security organizations and their impact on the meaning of life, using a mixed methodological orientation with an analysis of 93 members of the security forces. A non-experimental type of design of descriptive phenomenological effect was used, by providing, in an interview context, the Appreciation Questionnaire on Personal Role and Aspirations (CARAP) instruments and the Andrés Gottfried's Argentine Adaptation of the Purpose in Life Test (PIL TEST) of Crumbaugh and Maholick, in order to find out the extent of the meaning of life and social representations.

The findings show that $80 \%$ of the interviewees stated that they had their own idea of role: $17.20 \%$ were unaware of the duties and activities related to the role to be performed and the remaining $2.15 \%$ stated that they did not answer the question asked.

The results of the CARAP questionnaire and part B of the PIL Test enables us to affirm that it was possible to learn about the aspirations of the people who take part in security organizations. Likewise, it was evidenced that, in order to reach their aspirations in a significant percentage of $46.49 \%$, they acknowledge the need to make an effort, to give the best of themselves and to work to achieve what they long for. Finally, a proposal was elaborated to enrich the training processes of future applicants by receiving guidance with group dynamics and introducing them to consider the role they will play and the influence it will have on their meaning of life. 
Palabras Claves: Representaciones Sociales; Organización de Seguridad; Rol; Sentido de Vida; Psicología Social

Keywords: Social Representations; Security Organization; Role, Meaning of Life; Social Psychology 


\section{Introducción}

Dentro del ámbito elegido para llevar a cabo el trabajo se indagó acerca de la idea de rol y las aspiraciones personales de los participantes a fin de poder establecer relaciones entre las representaciones que manifiestan y la influencia que ejerce en relación con el sentido de vida.

El objetivo general fue describir las representaciones sociales de personas que forman parte de una organización de seguridad detallando su influencia respecto al sentido de vida.

Los objetivos específicos consistieron en analizar las representaciones sociales de personas que forman parte de una organización de seguridad; examinar el grado de realización de sentido de vida: establecer relaciones entre las representaciones sociales y el grado de realización de sentido de vida y conocer las aspiraciones personales de personas que forman parte de una organización de seguridad de seguridad

Sarbin y Vernon sustentan que la noción de rol dispone al hombre en un ambiente social definido y en un armazón social y grupal preciso. Del mismo modo enuncia que el intercambio entre personas se lleve a cabo dentro de una estructura grupal e intergrupal y eso requiere de un aprendizaje determinado de habilidades sociales para cada espacio social, que a su vez reflejan, los roles que toman los individuos en los diversos enfoques sociales (Aritzeta y Ayestarán, 2003).

Es Rodríguez Cueto (2008) quien argumenta en relación con los roles en grupos de trabajo, que son papeles o modos de proceder que los individuos asumen cuando se vinculan con otros. Algunos son instantáneos y otros son manifestación de facetas de personalidad y por ese motivo son considerados permanentes. Un integrante puede acoger más de un rol al mismo tiempo e ir alternando de rol en relación del progreso del equipo. De igual modo teniendo en cuenta las características 
de los individuos en el grupo se destacan los centrados en la tarea, los centrados en la subsistencia grupo y los negativos concentrados en conductas propias.

Los roles son el fruto de una construcción que se ha dado a través del tiempo y que está, institucionalmente establecida. La noción deriva del modelo dramático y transforma a los actores en personajes de un argumento compuesto para la conquista de los propósitos que se instalan. Asimismo, específica Goffman (1971) que la noción de rol organizacional es el que convierte al actor en personaje, diferenciando entre el retrato que una persona tiene de sí mismo, personaje, y su sustento material, físico, biológico, actor.

Frankl (1994) afirma que el sentido de vida comprende al propósito vital, los valores y la as respuestas que se proporcionan a los cuestionamientos que la vida plantea. Para el autor el sentido posee como cualidades el ser único, es decir, su Unicidad, ya que no hay dos sentidos iguales. El sentido es propio de cada Ser, y posee a su vez las cualidades de ser individual, intransferible y situado.

Precisa Martínez (2009) al sentido de vida como la percepción afectiva cognitiva de valores que estimulan al hombre a conducirse de una manera o de otra frente a circunstancias concretas o la vida en general suministrando cohesión e identidad personal. A la vez, Gottfried (2017) define el constructo sentido de vida aludiendo a que refiere a razones, inspiraciones en la vida y la frustración existencial como la carencia o falta de ese significado que mueve la voluntad del ser

La dimensión primordial unificadora y que enlaza lo cognitivo con lo emocional es la dimensión espiritual, al decir de Lanosa (2016). Agrega además que la espiritualidad se expresa a través de ciertas capacidades que enaltecen nuestro horizonte de conciencia para que podamos 
trascender. Las capacidades son el Auto-distanciamiento, la Autoreflexión, la Libertad, la Responsabilidad, la Autotrascendencia y la Autoconciencia. La primera alude a la facultad de poder apartarse de una situación y también de sí mismos; la Autorreflexión refiere a la facultad que nos permite razonar y pensar sobre situaciones que nos pertenecen y también sobre sí mismos; la Libertad nos permite adoptar una postura eligiendo en cada situación; la Responsabilidad está vinculada a la anterior y es la que nos brinda la posibilidad de responder a la elección que dimos con compromiso; La Autotrascendencia es la facultad de poder salir de nosotros mismos y encontrarnos con otro o dirigirnos a algo y la Autoconciencia es la capacidad de percibirnos como únicos e irrepetibles.

Moscovici definió a las representaciones sociales como un conjunto ordenado de conocimientos y una tarea psíquica a partir de la cual los seres humanos permiten que sea clara la realidad fisica y social, ya que, se incluyen en un grupo o vinculo periódico de intercambios en el cual sueltan el vigor de su imaginación. Refiere además que son un estilo específico de conocimiento, es decir, un modo determinado de descifrar la realidad, aludiendo al pensamiento del sentido común. El autor, Manifiesta además que las representaciones sociales responden a un desarrollo personal que supone una ordenación cognitiva pero que a la par halla su inicio, su difusión y cambio en el contexto sociocultural. Se destaca en esta dinámica la relación existente entre las representaciones sociales y la comunicación (Rodríguez Salazar y García Curiel, 2007).

Schvarstein (1991) establece una clara distinción de términos que son usados como sinónimos en la cotidianeidad detallando que no lo son, cuando se habla de organización, institución y establecimiento. En cuanto a la organización: menciona que son unidades compuestas y las distingue de las unidades simples que son como uno indiviso sin 
diferenciar las fracciones que lo conforman. Por otra parte, detalla que el interés se basa fundamentalmente en la interacción que se da entre las fracciones que componen la unidad y los individuos. Asimismo, acentúa la condición de construcción social que poseen las organizaciones, ya que, alude a un término cultural, convenido y que existe solo a través de la armazón que de él se hace y que como toda representación está en el lenguaje. Enuncia además que es un grupo organizado y estructurado de objetos tal cual los percibe el individuo, es decir una figura perceptiva o construcción perceptual. Al referirse a la institución; expresa que son organismos normativos jurídico-culturales formado por de pensamientos, valores, convicciones y legislaciones que determinan modos de intercambio social. La institución es un grado de realidad social que delimita lo que está determinado. Se vincula con el estado que pronuncia las leyes y desde esta perspectiva se encuentra inmerso en todos los grupos y organizaciones. Son desde esta concepción abstracciones, mientras que las organizaciones son su apoyo material. A modo de ejemplo alude a instituciones refiriéndose a entes tales como: la salud, la educación, el trabajo, la sexualidad, la religión, el tiempo libre. En cuanto a los establecimientos; enuncia que son aquellos a los cuales se les asigna un propósito social definido por una o más de las instituciones antes mencionadas. Sostiene además el autor, en relación con las organizaciones el hecho ineludible de estar atravesadas por muchas instituciones con las cuales se establece una relación que no tiene una única dirección, sino que se retroalimentan.

Losada, Rivela y Otero (2019) dieron nacimiento al constructo Organizaciones de Seguridad, pudiendo comprobar que hasta el momento de su investigación no había un término consensuado que permitiera agrupar a las fuerzas armadas, de seguridad y las instituciones civiles armadas bajo esta nominación, la cual consideran 
que el constructo es de suma relevancia para el campo de la psicología social

\section{Antecedentes}

Sirimarco (2009) detalla un trabajo de campo llevado a cabo con agentes de la academia de Suboficiales concernientes a policía federal de la Escuela Villar y Cadetes del instituto de formación de Policía perteneciente a la Provincia de Buenos Aires de la Escuela Juan Vucetich. Se realizaron en la academia Vucetich entrevistas a Cadetes en distintos momentos en los años, 2003 y 2004, y en la Escuela Villar fue llevado a cabo durante los años 1998 y 1999. Estas instituciones, presentan algunas particularidades que las diferencian dada la pertenencia institucional, las características de autoridad y subordinación. Por otra parte, presentan asimismo dinámicas de semejanzas en lo referido a las rutinas de instrucción. La autora Examino situaciones de campo antropológicas a través de un análisis etnográfico que le permitió analizar contribuciones acerca del rol y de las funciones que se exigen en las fuerzas de seguridad. Asimismo, sostiene que una investigación antropológica posee un alcance significativo pudiendo analizar $\mathrm{y}$ reflexionar sobre aquellos valores y sentidos que constituyen la experiencia policial y no son tenidos en cuenta en muchas ocasiones en otros tipos de análisis.

En la investigación de Bergman y Flom (2012) se pesquisa acerca de los determinantes de la confianza en la policía, comparando la percepción que los ciudadanos tienen acerca del desempeño de la policía tanto en México como en Argentina. El trabajo les permitió demostrar que la evaluación de desempeño policial es determinante de la confianza en la policía y para arribar a dicha afirmación elaboraron modelos de regresión 
logística con información de encuestas realizadas en la ciudad de Buenos Aires y en Distrito Federal. Las investigaciones han permitido asegurar que en ambos lugares es diferente la percepción, estando muy desvalorizada en Distrito Federal, México y siendo mejor considerada en Buenos Aires. De igual forma destacan que estas diferencias $y$ peculiaridades tienen que ver con la relación establecida históricamente entre la policía y las comunidades, estando presentes variables que influyen en la confianza en la fuerza policial tales como la clase social, el género, la edad. Sostienen además que los resultados de este estudio sugieren que antes de reprochar la denuncia de delitos y la confianza ciudadana, la policía debe aunar esfuerzos para mejorar su práctica.

Clavijo, Trincheri y Trincheri (2014) exponen un proyecto de trabajo conjunto en el cual analizan las representaciones sociales que poseen los jóvenes y policias en torno a conceptos tales como seguridad, justicia, victimas, orden y desorden vocación, derechos humanos y obediencia. El desarrollo del trabajo realizado permitió afirmar que se encuentran reiteraciones en los argumentos siendo mencionados de manera abierta y explicita al referirse a las prácticas policiales. Los autores arribaron a la conclusión de que extendiendo los procesos de formación y ofreciendo ámbitos distintos de aprendizaje sería posible lograr un mejor control de las praxis policiales.

El estudio de Díaz de Dragotta (2015) se halla vinculado con la agresividad y las psicopatias dentro de establecimientos de seguridad conducida desde la psiconeuroinmunoendocrinología, que es el estudio de las interacciones cerebro y sistema inmunológico y endocrinológico. Menciona como principal objetivo la indagación de parámetros que contribuyen al abordaje y tratamiento de la conducta agresiva. Esos elementos se congregaron abordando también el aspecto psicológico. La muestra comprende a 100 individuos que forman parte de la policía y 
también penitenciarios en la provincia de Mendoza. Los resultados obtenidos permitieron afirmar que los oficiales que habian sido denunciados por violencia familiar poseen variaciones en el eje corticohipotálamo-hipofisario-adrenal con compromiso neuroendocrino substancial. Por otra parte, los oficiales dedicados a labores de peligro evidenciaron rasgos de personalidad que dieron cuenta de una inadecuada expresividad e introversión siendo seres con indicadores de inmadurez emocional. Diaz de Dragotta (2015) acentúa que este abordaje permite valorar alteraciones en agentes en los que se presume de violencia o agresividad y de ese modo es posible prever su tratamiento. Describiendo además que las contribuciones más importantes de su trabajo guardan vinculación con las recurrencias que existe entre las señales de impulsividad observados en las técnicas del Test Gestáltico Visomotor y Rorschach con indicadores de las otras disciplinas evaluadoras lo que permite afirmar la necesidad del abordaje psico neuro inmune endocrinológico.

Losada $(2017,2019)$ manifiesta que el estrecho lazo que existe entre nociones y supuestos de la sociología de la salud y la psiconeuroinmunoendocrinología brindan convergencias y aspectos en común en el desarrollo de ambas áreas a partir de la elaboración de su conocimiento científico.

El término sociopsiconeuroinmunoendocrinología se atribuye por el predominio de la multideterminación del ambiente, de los vínculos sociales y de la cohesión social en los sistemas neurológico, endocrino e inmunitario que, a su vez, son influidos por la esfera psicológica. El paradigma neurocientífico imperante en este siglo da paso a la inclusión de esos sistemas, pero busca apuntar a su análisis desde una mirada integral de la salud. El estudio fue descriptivo a través los métodos de observación de documentos y una revisión crítica sistemática de 
literatura cientifica. Asimismo, propone futuras líneas de estudio, dejando abierta la posibilidad de abarcar otras perspectivas e incluir a investigadores de otras disciplinas en sus equipos de trabajo. La inclusión conocimientos proveniente de diversas ciencias pretende congregarse de modo tal que permita una concordancia dinámica.

Klos y Gutiérrez De Vázquez (2016) desarrollan un estudio en el cual abordan los valores y las motivaciones en relación con los estilos de liderazgo en cadetes que pertenecen a establecimientos de seguridad. La muestra se llevó a cabo con 210 individuos en formación en la academia de oficiales de Entre Ríos a los cuales se administraron cuestionarios de motivaciones ocupacionales-vocacionales; de valores y de estilos de liderazgo utilizando para su análisis la regresión lineal múltiple. Los resultados permitieron arribar a la conclusión de que tanto las motivaciones ocupacionales-vocacionales como los valores pueden predecir estilos de liderazgo. Por otra parte, expresan que conocer estas motivaciones y los valores brindan la oportunidad de seleccionar a los aspirantes y establecer roles en concordancia con el estilo que se aspira a desplegar.

Losada, Rivela y Otero (2019) presentaron una investigación en el cual mencionan como se llevó a cabo la construcción del Cuestionario de Apreciación sobre Rol y Aspiraciones Personales para ser administrado en personas que forman parte de una Organización de seguridad. E1 objetivo del trabajo aspiró a describir las ideas de rol y aspiraciones personales pudiendo realizar ajustes al instrumento y cambios en las variables consultadas a fin de arribar a un cuestionario definitivo. La muestra estuvo conformada por 33 participantes pertenecientes a fuerzas de seguridad, fuerzas armadas e instituciones civiles armadas. A partir de los aportes brindados por las personas entrevistadas lograron afinar el instrumento para ser administrado a futuro a un mayor número de 
personas que estén vinculadas en otros servicios de seguridad e incluir organizaciones como gendarmería y policía aeroportuaria. Los resultados obtenidos permitieron dar lugar al constructo Organizaciones de seguridad para designar, en adelante, bajo esta nominación a todas las fuerzas que las componen, es decir que al mencionar a las $\mathrm{OE}$ las autoras se están refiriendo a La Fuerza armada Nacional; compuesta por la Armada Argentina, Fuerza Aérea y Ejercito Argentino; Las Fuerzas de seguridad conformada por Gendarmeria, Prefectura Naval Argentina, Policía de Seguridad Aeroportuaria y Policía Federal Argentina: Las Instituciones Civiles Armadas integradas por Policía de la Ciudad Autónoma de Buenos Aires, Policía de la Provincia de Buenos Aires, Policía de las Provincias y Unidad de Policía de Prevención Local. Asimismo, destacan que no están incluidos dentro de este constructo servicios como Bomberos voluntarios, seguridad privada de bienes y personas y servicio penitenciario por no compartir funciones y misiones específicas en relación con la defensa nacional y seguridad interior específicamente.

La iniciativa que impulsó este estudio de indagar las representaciones sociales y aspiraciones personales de personas que forman parte de organizaciones de seguridad detallando su influencia en el sentido de vida surgió a partir de considerar a la psicología social como una disciplina que centro su interés en las relaciones interpersonales. Dada su particular característico de ser considerada una disciplina fronteriza, la perspectiva que promueve resulta un instrumento útil, además, para expertos de otras áreas (Ovejero Bernal, 2007).

Perlan y Crosby sostienen que los procesos esenciales para comprender como alcanzamos a conocer y vincularnos con los demás, son centrales para adentrase en la comprensión de las relaciones sociales. Por este motivo se considera que describir las ideas y 
representaciones de las personas permite profundizar en la realidad que está reunida en la mente bajo una estructura cognitiva, y que, a su vez, simboliza un conocimiento ordenado acerca de algo (Ovejero Bernal, 2007).

Por otra parte, investigar el contexto de la organización resulta un campo predilecto para obtener información que ilustre las manifestaciones sociales y poder ahondar en la comprensión y análisis de aspectos psicosociales que en ellas se gestan, aspectos que, están en consonancia con la realidad (Peiro, 1990).

A respecto de la realidad Berger y Luckmann conciben que la realidad de la vida diaria es una condición de los fenómenos que se muestran sin estar ligados a la voluntad y el conocimiento y sin que sea necesario adjuntar otras comprobaciones, la realidad es tal cual se presenta (Porras Velásquez, 2012).

Por lo expuesto se investigó en torno a la posibilidad de conocer las representaciones sociales en organizaciones de seguridad y establecer vínculos con el sentido de vida en el marco de la psicología social con la pretensión de poder analizar las ideas que forman parte del imaginario de las personas pertenecientes a esta realidad y establecer relaciones significativas entre esas imágenes mentales y el modo que influyen en su sentido de vida.

\section{Materiales y métodos}

El estudio se llevó a cabo con una orientación cuanti- cualitativo siendo la muestra de clase no probabilística de tipo homogénea.

Teniendo en cuenta a Hernández Sampieri, Fernández Collado y Baptista Lucio (2010) los enfoques mixtos son la integración sistemática de los métodos cuanti y cualitativos en uno solo. Son estudios que tienen 
como finalidad obtener una fotografia más completa del fenómeno pudiendo combinar al menos un componente cuantitativo y uno cualitativo en un mismo estudio o proyecto de investigación.

El tipo de diseño que se utilizó fue no experimental de alcance fenomenológico descriptivo. Hernández Sampieri, et al. (2010) consideran no experimental porque su finalidad es estudiar el fenómeno del modo que se presenta en su entorno natural para poder examinarlo. Por otra parte, la investigación es transeccional descriptiva dado que tiene como propósito congregar información en un solo momento para poder indagar la incidencia de particularidades y características propias del grupo de análisis y arribar a su descripción.

De acuerdo con los principios éticos de la investigación se utilizó el consentimiento informado dado que el mismo se constituye como un instrumento que permite ser utilizado para la protección de los derechos de los participantes (Losada, 2014).

El análisis cuantitativo se realizó mediante observación sistemática. Según Piñar, Caro y Coscollá este tipo de observación se realiza utilizando código arbitrario de observación que será construido con antelación y cuyo motivo será descriptivo (Montero y León, 2007).

La unidad de muestreo se conformó con una población de 93 miembros de Organizaciones de Seguridad, específicamente integrantes de las fuerzas armadas, fuerzas de seguridad, instituciones civiles armada y servicios de seguridad residentes de la Ciudad Autónoma de Buenos Aires, Gran Buenos Aires y Costa Atlántica, Formosa y Tucumán, de la República Argentina. La muestra ha sido de clase no probabilística intencional de tipo homogénea. Fassio, et al. (2004) al respecto manifiestan que esta clase es valorada de este modo debido a que son muestras en las que se desconoce la probabilidad que tiene cada 
participante de ser incluido. La elección obedeció a motivos vinculados con las particularidades de la investigación.

Los participantes miembros de Organizaciones de Seguridad que conformaron la unidad de análisis son personas de edades comprendidas entre 26 a 79 años de edad de ambos sexos residentes en Argentina, 59 de los participantes son varones siendo el 63,44\% y los 34 restantes mujeres conformando el 36,56\%. De los 93 colaboradores: 7, manifestaron tener una edad comprendida entre 26 a 29 años de edad siendo el 7,53\%; 45, entre 30 y 39 años siendo el rango más significativo con un porcentaje de 48,39\%; 20, entre 40 y 49 años siendo el 21,51\%; 4, entre 50 y 59 años siendo la población minoritaria de la muestra con un 4,30\%; 12, entre 60 y 69 años siendo el 12,90\%; y los 4 restantes, entre 70 y 79 años siendo el 5,38\%.

En relación a la antigüedad desempeñado el rol se pudo apreciar que 34 participantes poseen hasta 5 años siendo el 36,56 \%; 16, entre 6 a 10 años siendo el 17,20\%; 20, entre 11 a 20 años comprendiendo el 21,51\%, 11 personas entre 21 a 30 años desempeñando tareas y prestando servicio siendo el 11,83\% y los 12 restantes expresaron llevar más de 30 años trabajando en la organización a la que pertenecen siendo el 12,90\%.

En relación a la capacitación y niveles de estudios alcanzados 2 participantes, culminaron el nivel primario siendo el 2,15\%; 48, el nivel secundario siendo el 51,61\%; 28, finalizaron estudios terciarios siendo el $30,11 \%$ y los 15 participantes restantes estudios universitarios siendo el 16,13\%. De los 93 participantes; 9 personas , pertenecen a la Armada Argentina siendo el 9,68\%; 1, al Ejército Argentino siendo el 1,08\%; 5, a la Fuerza Aérea Argentina siendo el 5,38\%; 1, a Gendarmería Nacional siendo el 1,08\%; 7, a la Policía de la Ciudad Autónoma de Buenos Aires siendo el 7,53\%; 5, a la policía de Tucumán siendo el 5,38\%; 1, Policía 
de seguridad Aeroportuaria siendo el 1,08\%; 20, a Policía Federal siendo el 221,51\%; 6 policía Local de la Provincia de Buenos Aires siendo el 6,45\%; 33, a Policía de la Provincia de buenos Aires siendo el 35,48\% y la organización de seguridad con mayor participantes de la muestra ; los 4 participantes restantes pertenecen a servicios de seguridad, seguridad privada 1, siendo el 1,08\%, 1, bomberos siendo el 1,08\% y 2 , penitenciarios siendo el $2,15 \%$.

Los instrumentos utilizados para recabar información fueron la entrevista, en la cual, se administró a los participantes el Cuestionario de apreciación sobre Rol y Aspiraciones Personales -CARAP- y la adaptación argentina de Andrés Gottfried del Purpose in Life Test -PIL TEST- de Crumbaugh y Maholick.

\section{Resultados}

El objetivo principal de este trabajo ha sido describir las representaciones sociales de personas que forman parte de organizaciones de seguridad y conocer si ha existido influencia de las mismas en su sentido de vida. Para ello primero se obtuvieron los datos de 93 personas miembros organizaciones de seguridad. Del objetivo principal se desprendió la pregunta de investigación ¿Las representaciones Sociales de personas que forman parte de una organización de Seguridad influyen en su sentido de vida?

Se realizó un relevamiento de las respuestas dadas por los participantes y los hallazgos permiten afirmar que si es posible describir las representaciones sociales que poseen.

Los resultados obtenidos revelan que las representaciones sociales de personas que forman parte de una organización influyen en su sentido de vida. Fue posible describir la idea de Rol, es decir la representación 
social de rol de los participantes antes de ingresar a la formación. De las 96 personas entrevistadas, 75, manifestaron tener su propia idea de rol siendo el 80, $65 \%$; 16, desconocian las tareas y actividades relacionadas con el rol a desempeñar siendo el 17,20\%; y los 2 participantes restantes dieron respuestas que no respondian a la pregunta realizada siendo el 2,15\%; Asimismo se logran describir la motivaciones para ingresar a la formación que poseen las personas que forman parte de una organización; se pudo observar que de los 93 participantes, 88 seleccionaron una sola motivación, lo que equivale a un $94,62 \%$ y los 5 restantes mencionaron 2 motivaciones, lo que equivale a un $5,38 \%$. Se evidencia que una de las dos motivaciones fue la misma para "salida laboral" y la otra fue diferente para los 5 participantes; 2 seleccionaron "motivos familiares" y "salida laboral"; 1 participante selecciono "salida laboral y vocación"; 1 participante selecciono "para ayudar a los demás" y "salida laboral", 1 participante selecciono "salida laboral" y "por crecimiento personal y profesional"; Se detalla el análisis en relación a las motivaciones que expresaron: no manifestó tener ninguna razón en particular 1 respuesta siendo el 1, 02\%; explicitó que no correspondía la pregunta según su situación 1 respuestas siendo el 1,02\% ; sintió la obligación de hacerlo 1 respuesta siendo el 1,02; por respeto a la fuerza 1 respuesta siendo el 1,02\%; por curiosidad 4 respuestas siendo el 4,08\% ; para desarrollar otras profesiones dentro de la fuerza pudiendo luego capacitarse y acceder a otras 4 respuestas siendo el 4,08\% ; por crecimiento personal y profesional 4 respuestas siendo el 4,08\% ; por insistencia de familiares y amigos 5 respuestas siendo el 5, 10\%; por gusto 7 respuestas siendo el 7, 14\%; para ayudar a los demás 9 respuestas siendo el 9,18\% ; por razones tuvieron que ver con motivos familiares e identificación con padres o hermanos en la 
fuerza 16 respuestas siendo el 16, 33\% ; por vocación 20 respuestas siendo el 20, 41\% y por salida laboral 25 respuestas siendo el 25,51\%..

Otro Hallazgo que se detalla en cuanto a si la motivación cambió o se mantuvo mientras permanecían en formación permitiendo evidenciar, dados los resultados obtenidos, que la elección de elegir capacitarse y ser parte de una organización tuvo influencia en su sentido de vida. De los 93 participantes, 1 no responde a la pregunta siendo el 1,08\%; 3 expresaron que sólo cambió -ni aumento ni disminuyo- siendo el 3,23\%; 9 sintieron que su motivación fue disminuyendo por no ser lo que se imaginaban y por haber cambiado sus expectativas durante la formación siendo el 9, 68\%; 29 expresaron que la motivación fue creciendo y superaron sus expectativas siendo el 31, 18\%; y el 51 restante mantuvo la motivación por la cual ingreso siendo el 54, 84\%.

De los objetivos específicos de la presente investigación se desprenden las preguntas de trabajo nominadas como 2, 3 y 4. Los hallazgos que se expresan en los resultados permiten afirmar que se han podido comprobar en su totalidad. Del objetivo de analizar las representaciones sociales de personas que forman parte de una organización, se desprende la pregunta de investigación 2: ¿En posible analizar las representaciones sociales de personas que forman parte de una organización de seguridad? Los hallazgos obtenidos permiten afirmar que se ha podido cumplimentar, dado que, los resultados obtenidos pudiendo establecer un análisis exhaustivo en relación a las aspiraciones personales de los sujetos que son las representaciones sociales que poseen de esas aspiraciones.

Para el análisis se tomó la decisión metodológica de vincular las aptitudes y apreciaciones con las capacidades espirituales que concibe la logoterapia. La cantidad de representaciones sobre aptitudes expresadas 
fue de 67 y se evidencio que los participantes en muchos casos mencionaron más de una aptitud. En la tabla $N^{\circ} 9$ se detalla la cantidad de participantes que mencionaron entre 1 a 7 aptitudes en sus respuestas. 32 Participantes expresaron 1 aptitud en sus respuestas siendo el 34, 41\%; 25 personas mencionaron 2 aptitudes siendo el 26,88\%; 20, 3 aptitudes siendo el 21, 51\%; 9 colaboradores, 4 aptitudes siendo el 9,68\%; 5, expresaron 5 aptitudes siendo el 5, 38\%; 1 participante expreso 6 siendo el 1, 08\%; y el restante expreso 7 diferentes aptitudes siendo el 1,08\% restante.

De los 93 participantes: 14 de ellos que corresponde al 6,60\% menciono relevante la aptitud física como condición para formar parte de una organización de seguridad, 3 que corresponde al 1,42\% aptitud psicológica; 5 que corresponde a 2,36\% expresa que ser capaz es la condición que consideran; 3 que corresponde a 1,42\% ser ético; 1 que corresponde a 0,47\% íntegro; 2 que corresponde al 0,94\% poseer sentido de pertenencia; 1 que corresponde a 0,47 ser resiliente. 1 que corresponde a $\mathrm{El} \mathrm{0,47 \%} \mathrm{de} \mathrm{los} \mathrm{participantes} \mathrm{respondió} \mathrm{que} \mathrm{ser} \mathrm{firme} \mathrm{es}$ condición necesaria; 1 que corresponde a $0,47 \%$ proactivo; 6 que corresponde a 2,83 \% resolutivo; 2 que corresponde a $0,94 \%$ afirmo que el temple es fundamental como condición; 3 que corresponde a 1,42\% tolerante; 3 que corresponde a 1,42\% valiente; 1 que corresponde a $0,47 \%$ expreso que el autocontrol es indispensable como aptitud; 1 que corresponde a $0,47 \%$ el sentido común; 1 que corresponde a $0,47 \%$ ser una persona de pocas palabras; 2 que corresponde a 0,94 mantenerse equilibrado; 2 que corresponde a 0,94 destaco la inteligencia y 2 que corresponde a 0,94\% mostrarse seguro. 1 que corresponde al 0,47\% menciono que el altruismo es importante ; 1 que corresponde al 0,47\% ser benevolente; 1 que corresponde a 0,47\% ser bondadoso;7 que corresponde a $3,30 \%$ ser camarada y compañero con los demás; ser 
compasivo 1 que corresponde a $0,47 \% ; 14$ tener vocación que corresponde a $6,60 \% ; 2$ poder cooperar que corresponde a $0,94 \% ; 5$ ser empático que corresponde a 2,36\%; 1 menciono la fidelidad que corresponde a $0,47 \% ; 1$ expreso la generosidad que corresponde a 0,47\%; 11 ser honesto que corresponde a 5, 19\%,3 mostrarse honorable siendo el 1,42\%; 6 la lealtad siendo el 2,83\%; 8 ser servicial siendo el 3, 77\%; 2 sincero siendo el 0,94\% ; 5 solidario siendo el 2,36\%; 3 que corresponde al 1,42\% manifestó que la aptitud guardaba relación con ser comunicativo; 2 que corresponde al 0,94\% ser educado; 2 que corresponde al $0,94 \%$ ser espiritual; 1 que corresponde al 0,47\% estructurado; 2 que corresponde al 0,94\% menciono ser fortalecido; 3 que corresponde al 1,42\% honrado; 5 que corresponde al 2,36\% humilde; 1 que corresponde al 0,47\% tener capacidad para ser líder; 3 que corresponde al 1,42\% paciente; 1 que corresponde al $0,47 \%$ mantener un perfil bajo; 3 que corresponde al 1,42\% tener predisposición;2 que corresponde al $0,94 \%$ de aspecto presentable; 1 que corresponde al 0,47\% recto; 13 que corresponde al 6,13\% respetuoso; 1 que corresponde al 0,47\% sacrificado; 1 que corresponde al 0,47\%; 4 que corresponde al 0,47\% sereno ; 4 que corresponde al 1,89\% serio; 1 que corresponde al 0,47\% sumiso. 10 que corresponden al 4,72\% menciono relevante la capacitación y el adiestramiento; 4 que corresponden al $1,89 \%$ tener compromiso; 1 persona que corresponde al $0,47 \%$ ser criterioso; 2 personas que corresponden al 0,94\% cuidadoso; 1que corresponde al 0,47\% actuar con decoro; 1 que corresponde a 0,47\% mostrarse dedicado; 2 que corresponde al 0,94\% con disciplina; 1 que corresponde al 0,47\% eficiente; 1 que corresponde al 0,47\% justo; 1 que corresponde al $0,47 \%$ obediente; 2 que corresponde al 0,94 perseverante; 5 que corresponde al 2,36\% profesional; 8 que corresponde 
al $3,77 \%$ ser responsable; y 1 que corresponde al 0,47\% restante menciono que debe presentar aptitudes relacionadas con ser trabajador.

Se han podido evidenciar las capacidades que estuvieron presentes de acuerdo a las 67 aptitudes manifestadas y la cantidad de respuestas dadas por cada participante y su correspondiente porcentaje.

Con respecto al total de 93 participantes, dieron 69 respuestas que corresponden a autotrascendencia el 32, 55\%; 49 el 23,11\% con la libertad; $18,87 \%$ con la responsabilidad; 13,68 con la autoconciencia; 7 , $55 \%$ con el autodistanciamiento y el 4, 25\% restante con el autorreflexión.

Acerca de las apreciaciones manifestadas sobre la función que desempeñan pudo evidenciarse que los de los 93 participantes, 72 mencionaron una apreciación lo que equivale a un $77,42 \% ; 16$ mencionaron dos apreciaciones siendo el 17,20\%; 3, manifestaron 3 opiniones respecto de su actividad siendo el 3,23\% y los 2 restantes expresaron 4 apreciaciones que equivale a un 2, $15 \%$.

En cuanto a los resultados sobre las capacidades espirituales que se evidenciaron, la cantidad de respuestas que dieron los participantes y el porcentaje que corresponde a cada una. El 54,55\% que corresponde a 66 respuesta dada coincide con autorreflexión; el 23,14\% que corresponde a 28 respuestas dadas coincide con a la capacidad de autotrascendencia; el 18,18\% que corresponde a 22 respuestas dadas coincide con a la autoconciencia y el 4, 13\% que corresponde a 5 respuestas dadas coincide con la capacidad de la libertad. No estuvieron presentes ideas y representaciones sobre la actividad que den cuenta de las capacidades de autodistanciamiento y responsabilidad.

Se congregaron los resultados asociados a la portación de arma de los 93 participantes; 55 manifestaron que la particularidad de tener que 
portar arma no les influyo en su vida, lo vivencian como parte del trabajo y de pertenecer a la organización siendo el 59,14\%; 8 personas expresaron que por su condición de ser administrativos no portan arma siendo el 8,60\% y 30 sujetos respondieron que si les influyo la portación siendo el 32,26\%.

Con respecto al objetivo de examinar el grado de realización de sentido de vida, se ha desprendido la pregunta ¿Cuál es el grado de realización de sentido de vida de personas que forman parte de una organización de seguridad? Ha sido posible cumplimentar y determinar el grado de realización de sentido de vida de personas que forman parte de organizaciones de seguridad. Los resultados obtenidos evidencian en la muestra de 93 participantes pertenecientes a organizaciones de seguridad. De los 93 participantes, 7 de ellos, el 7,53\% del total de la muestra, obtuvieron un puntaje igual o menor al percentil 10 siendo muy bajo y correspondiéndose a Neurosis Noógena; 25 personas, el $26,88 \%$,obtuvieron un puntaje igual o menor al percentil 25 siendo bajo y correspondiendo a vacío existencial; 45, el 48,39\%, obtuvieron puntajes entre percentil 26 y el percentil 40 siendo promedio bajo y correspondiéndose a zona de frustración: alarma y riesgo; 13 , el 13,98\%, obtuvieron puntajes entre el percentil 41 y el 60 siendo promedio y correspondiéndose a zona de indefinición de sentido y los restantes, el $3,23 \%$ obtuvieron puntajes entre el percentil 61 y el percentil 74 siendo promedio alto y correspondiéndose con orientado al logro de sentido.

Con respecto al objetivo de establecer relaciones entre las representaciones sociales y el grado de realización de sentido de vida se ha desprendido la pregunta de investigación: ¿Se pueden establecer relaciones entre las representaciones sociales y el grado de realización de sentido de vida? Los resultados obtenidos permiten afirmar que es posible establecer correspondencias significativas entre las 
representaciones sociales de personas que forman parte de organizaciones de seguridad y el grado de realización de su sentido de vida

Los hallazgos obtenidos permiten confirmar que las aptitudes expresadas por los participantes respecto a: lo que consideran que debe tener una persona para formar parte de una organización; las apreciaciones dadas sobre su función y actividad; y la apreciación sobre la portación de arma, dan cuenta de la idea y representación que poseen. Por ello es posible afirmar que están presentes las representaciones sociales sobre rol en los hallazgos.

Observando los resultados en relación con el grado de sentido de vida se ha podido comprobar que inciden significativamente. Para el análisis se tomó la decisión metodológica de vincular las aptitudes y apreciaciones con las capacidades espirituales que concibe la logoterapia. De los 93 participantes: 14 de ellos que corresponde al 6,60\% menciono relevante la aptitud física como condición para formar parte de una organización de seguridad, 3 que corresponde al 1,42\% aptitud psicológica; 5 que corresponde a 2,36\% expresa que ser capaz es la condición que consideran; 3 que corresponde a 1,42\% ser ético; 1 que corresponde a 0,47\% integro; 2 que corresponde al 0,94\% poseer sentido de pertenencia; 1 que corresponde a 0,47 ser resiliente. 1 que corresponde a $\mathrm{El}$ 0,47 \% de los participantes respondió que ser firme es condición necesaria; 1 que corresponde a $0,47 \%$ proactivo; 6 que corresponde a 2,83 \% resolutivo; 2 que corresponde a $0,94 \%$ afirmo que el temple es fundamental como condición; 3 que corresponde a 1,42\% tolerante; 3 que corresponde a 1,42\% valiente; 1 que corresponde a 0,47\% expreso que el autocontrol es indispensable como aptitud; 1 que corresponde a $0,47 \%$ el sentido común; 1 que corresponde a $0,47 \%$ ser una persona de pocas palabras; 2 que corresponde a 0,94 mantenerse 
equilibrado; 2 que corresponde a 0,94 destaco la inteligencia y 2 que corresponde a $0,94 \%$ mostrarse seguro. 1 que corresponde al 0,47\% menciono que el altruismo es importante ; 1 que corresponde al 0,47\% ser benevolente; 1 que corresponde a 0,47\% ser bondadoso;7 que corresponde a 3,30\% ser camarada y compañero con los demás; ser compasivo 1 que corresponde a $0,47 \% ; 14$ tener vocación que corresponde a 6,60\%; 2 poder cooperar que corresponde a $0,94 \% ; 5$ ser empático que corresponde a 2,36\%; 1 menciono la fidelidad que corresponde a $0,47 \% ; 1$ expreso la generosidad que corresponde a 0,47\%; 11 ser honesto que corresponde a 5, 19\%,3 mostrarse honorable siendo el 1,42\%; 6 la lealtad siendo el 2,83\%; 8 ser servicial siendo el 3, $77 \% ; 2$ sincero siendo el 0,94\%; 5 solidario siendo el 2,36\%; 3 que corresponde al 1,42\% manifestó que la aptitud guardaba relación con ser comunicativo; 2 que corresponde al 0,94\% ser educado; 2 que corresponde al $0,94 \%$ ser espiritual; 1 que corresponde al $0,47 \%$ estructurado; 2 que corresponde al 0,94\% menciono ser fortalecido; 3 que corresponde al 1,42\% honrado; 5 que corresponde al 2,36\% humilde; 1 que corresponde al 0,47\% tener capacidad para ser líder; 3 que corresponde al $1,42 \%$ paciente; 1 que corresponde al $0,47 \%$ mantener un perfil bajo; 3 que corresponde al 1,42\% tener predisposición;2 que corresponde al 0,94\% de aspecto presentable; 1 que corresponde al $0,47 \%$ recto; 13 que corresponde al $6,13 \%$ respetuoso; 1 que corresponde al 0,47\% sacrificado; 1 que corresponde al 0,47\%; 4 que corresponde al 0,47\% sereno ; 4 que corresponde al 1,89\% serio; 1 que corresponde al 0,47\% sumiso. 10 que corresponden al 4,72\% menciono relevante la capacitación y el adiestramiento; 4 que corresponden al $1,89 \%$ tener compromiso; 1 persona que corresponde al $0,47 \%$ ser criterioso; 2 personas que corresponden al 0,94\% cuidadoso; 1que corresponde al $0,47 \%$ actuar con decoro; 1 que corresponde a $0,47 \%$ 
mostrarse dedicado; 2 que corresponde al 0,94\% con disciplina; 1 que corresponde al 0,47\% eficiente; 1 que corresponde al $0,47 \%$ justo; 1 que corresponde al $0,47 \%$ obediente; 2 que corresponde al 0,94 perseverante; 5 que corresponde al 2,36\% profesional;8 que corresponde al $3,77 \%$ ser responsable; y 1 que corresponde al $0,47 \%$ restante menciono que debe presentar aptitudes relacionadas con ser trabajador.

Se tomó la decisión de vincular una pregunta del cuestionario CARAP con las capacidades espirituales las primeras 11 respuestas de la tabla 13 coinciden con la capacidad de autoconciencia: Aprender diariamente corresponde a 4 respuestas dadas por los participantes siendo el 3,31\% ; 1 el riesgo físico siendo el 0,83\%; ; 1 riesgo psicológico siendo el 0,83\%; 3 la necesidad de adquirir conocimientos siendo el 2,48\%; 1 cuidar de sí mismo siendo el $0,83 \% ; 1$ capacitarse y actualizarse 0,83\%; 7 ser responsable $5,79 \%$; 1 ser consciente el 0,83\%;1 poseer inteligencia 0,83\%; 1 tener preparación física 0,83\%: 1 considerar la tarea como un desafío permanente $0,83 \% ; 1$ actividad desvirtuada lo considera el 0 , $83 \%$; 3 labor rutinaria 2,48\%; 1 comprometida 0,83\%;1 correcta 0,83\%; 3 desgastante 2,48\%; 1 esencial 0,83\%, 1 hermosa 0,83\%; 1 honesta 0,83\%;1 honorable $0,83 \% ; 1$ motivadora $0,83 \% ; 1$ muy buena $0,83 \% ; 1$ sacrificada $0,83 \% ; \quad 2$ satisfactoria $1,65 \% ; 1$ tranquila $0,83 \% 4$ considerada un trabajo más 3,31\%; 7 como un trabajo muy importante 5,79\%;3 útil 2,48\%; 1valorada $0,83 \% ; 1$ mal pagada 0,83\%; 1donde se hace o correcto $0,83 \%$ que causa orgullo $2,48 \%$; 4 que da gusto $3,31 \%$; 1 que no es lo que pensaban que era $0,83 \%$; 1 donde no se brindan oportunidades de mostrar los conocimientos $0,83 \% ; 4$ donde faltan medios y recursos 3,31\%; 1 pocas veces gratificante $0,83 \%, 4$ poco reconocida y valorada 3,31\%; 2 donde no se está protegido 1,65\%; 1 requiere ser dedicado $0,83 \% ; 1$ ser profesional $0,83 \% ; 1$ no se posee respaldo $0,83 \% ; 1$ se debe tener sentido común $0,83 \% 3$ requiere tener 
vocación $2,48 \%$; 1 se tienen buenos jefes $0,83 \% 2$ se puede trabajar adecuadamente 1,65\%; 2Sienten amor por su trabajo el 1,65\%; 2 les permite apoyar a sus compañeros el 1,65\%; 2 que superó las expectativas y aspiraciones el 1,65\%; 1 pudo desplegar su especialidad el 0,83\%; 16 que se siente conforme y a gusto el 13, 22\%; 3 su actividad le permite la prevención y poder velar por el otro el 2,48\%; 1que le permite mejorar el $0,83 \% ; 1$ ser empático 0,83\%; 5 que corresponde a el 4,13\% de los participantes no respondió a esta pregunta.

Otro hallazgo relevante obtenidos fue que de los 93 participantes, 7 de ellos, el 7,53\% del total de la muestra, obtuvieron un puntaje igual o menor al percentil 10 siendo muy bajo y correspondiéndose a Neurosis Noógena; 25 personas, el 26,88\% ,obtuvieron un puntaje igual o menor al percentil 25 siendo bajo y correspondiendo a vacío existencial; 45, el 48,39\%, obtuvieron puntajes entre percentil 26 y el percentil 40 siendo promedio bajo y correspondiéndose a zona de frustración: alarma y riesgo; 13 , el 13,98\%, obtuvieron puntajes entre el percentil 41 y el 60 siendo promedio y correspondiéndose a zona de indefinición de sentido y los restantes, el 3,23\% obtuvieron puntajes entre el percentil 61 y el percentil 74 siendo promedio alto y correspondiéndose con orientado al logro de sentido.

\section{Discusión}

En esta investigación se desarrolló una descripción de las representaciones sociales en organizaciones de seguridad en relación con la influencia que ejerce en su sentido de vida.

En este apartado se describen las conclusiones de los resultados obtenidos en este estudio relacionándolos con otras investigaciones afines desde la perspectiva teórica. Martínez (2009) brinda una definición 
de sentido que incluye a los valores y a la apreciación emotiva y cognitiva que cada persona posee. En línea con el autor fue posible demostrar que

Tanto las aspiraciones personales como la representación social sobre el rol que poseen las personas que forman parte de una Organización de Seguridad influyen en la realización del sentido de vida, dado que, esas ideas son inseparables de la escala de valores que las sustentan y lo que sienten, aprecian, consideran o creen es lo que valoran como prioritario para llevar a cabo sus propósitos en la vida. El análisis de los datos obtenidos permite corroborar dicha afirmación. Efectivamente los datos obtenidos muestran una clara relación entre las aspiraciones personales, ideas y representaciones sociales de rol y el grado de realización e influencia que ejercen en el sentido de vida en personas que forman parte de una organización de seguridad.

En concordancia con diversos autores que sostienen que el término organización alude a relaciones entre personas que establecen unidades sociales complejas distinguiéndose de otras simples por ser un grupo organizado, reglamentado por pautas roles y funciones determinadas orientadas a lograr fines específicos teniendo en cuenta la división de labores, responsabilidades, jerarquías, comunicación y figuras que son ejes de poder es posible afirmar que las personas que componen la muestra de esta investigación y que forman parte de diferentes fuerzas de seguridad componen una organización. Se utilizó la noción de organizaciones de seguridad en esta investigación para referirse a través de un solo termino a todas las fuerzas que están a cargo de la seguridad interior y de defensa nacional en línea con la investigación de Losada Rivela y Otero (2019) el constructor organizaciones de seguridad es de suma relevancia, dado que, hasta el momento no existía un término consensuado que permitiese agrupar a las fuerzas armadas, de seguridad y las instituciones civiles armadas bajo esta nominación. 
Las ideas reveladas en relación al rol, la motivación de ingreso y sus cambios durante la formación no arrojan relación con el género, los estudios cursados o la edad, es decir, que lo objetado por los participantes fue diverso independientemente de ser hombres, mujeres o el rango etario en el cual se encontraban.

La recopilación de aportes y el recorrido que se ha realizado por el pensamiento antropológico de los autores presentes en este trabajo ha tenido el anhelo de desarrollar un marco de conceptos y estudios que permitan explorar las representaciones sociales desde la mirada de expertos en el tema como así también algunas de las contribuciones esenciales en torno a la logoterapia y el sentido de vida.

Se considera que abordar a la persona humana desde la mirada integral, requiere ineludiblemente de la interdisciplinar, cada disciplina puede conocer tan solo una parte del ser, pero el trabajo conjunto entre ellas permite acercarse un poco más y emprender el desafio de comenzar a comprenderlo. Cada época posee un paradigma que impera y no es ajeno a ello la mirada de mundo y de individuo a la cual se adhiera.

Desde el nacimiento se es parte de una sociedad, es sabido que un recién nacido bebe no puede sobrevivir si no es gracias a otro, que lo alimenta, lo mira, lo contiene, y va descifrando lo que le sucede para brindarle lo que necesita. A medida que vamos creciendo vamos procurando hacer vínculos nuevos, e integramos una familia, un grupo de compañeros de escuela, de amigos, de deportes, también aprendemos códigos sociales, normas, leyes y de todo ello se posee una imagen, es decir, una representación. Esa es la realidad percibida y se tiene una representación de esa realidad también.

Desde pequeños se aprende acerca del sentido que será el que guie nuestro existir. 
Las representaciones que se posean, las que se compartan con el entorno serán parte de la vida, una vida que es social y que necesita de individualidad, para aportar a lo colectivo. Considerando que la solidaridad es poder trascender, poder salir de uno mismo al encuentro con otros para transitar la vida. Los hechos sociales, son los que se vive acompañados de otros y con quienes se comparte el compromiso de forjar un mundo mejor.

Las consideraciones que fueron incluidas en este trabajo tienen la pretensión de abrir un espacio de dialogo, que permita poder reflexionar acerca de la persona, de sus representaciones y del sentido que tiene su existir.

Se es persona, se tiene un cuerpo, que manifiesta lo que nos pasa y como nos sentimos, un organismo que tiene necesidades biológicas pero que también tiene una vida psíquica con representaciones conscientes e inconscientes, un espíritu que nunca enferma y que le permite trascender superando hasta las situaciones más adversas porque posee capacidades únicas y además se es parte de una sociedad a la cual debemos salir al encuentro.

\section{Conclusiones}

La concepción que plantea el presente trabajo sobre las organizaciones de seguridad enriquece el marco conceptual existente hasta el momento y permite establecer una amplitud en el término al vincularlo a la seguridad tanto interior como de defensa del territorio.

La psicología social entendida como tal no puede prescindir de la intervención de la política, las instituciones, organizaciones, aspiraciones personales, roles que se desempeñan y la influencia que ejerce el individuo en los grupos y sociedades a las que pertenece No puede 
hacerlo porque son parte de ella, la constituyen como tal. Desde este punto de vista se considera un gran aporte al campo de la psicología social el abordaje de las representaciones sociales y aspiraciones personales de las personas que forman parte de organizaciones de seguridad, dado que, permite un acercamiento para poder iniciarse en la comprensión de los fenómenos que acontecen en la realidad de estas organizaciones.

Una limitación que posee el presente estudio es que no abarcó las Organizaciones de Seguridad de todas las provincias del territorio argentino. Para que estos resultados sean generalizados sería necesario ampliar la muestra con un mayor número de participantes provenientes de organizaciones de todo el país y también dejando abierta la posibilidad a llevarla a cabo en otros países. Otra limitación externa fue la insuficiente investigación existente que comprenda todas las organizaciones en nuestro país.

Se evidenciaron inseguridades y cierta resistencia manifestada en muchas personas que forman parte de organizaciones de seguridad no permitiendo realizar una muestra que representara más sujetos de cada fuerza.

El trabajo precedente está sustentado en bases teóricas y antropológicas solidas siendo un aporte muy valioso como herramienta para psicólogos sociales que se desempeñen en organizaciones. La visión integral de persona permite complementar la labor de colegas, ya que considera al ser desde lo biológico, psicológico, social y espiritual.

El cuestionario CARAP resulta una valiosa herramienta para poder ser administrada por profesionales de la salud a personas que aspira ingresar a la formación en organizaciones de seguridad. Las preguntas permiten conocer las aspiraciones e ideas que los postulantes poseen 
sobre el rol y también al grado de realización de su sentido de vida hasta ese momento.

La adaptación Argentina del PIL test permite conocer el grado de realización de sentido de vida, lo cual, resulta apropiado para conocer si está orientada a su sentido o no. La relación de ambos instrumentos es un punto fuerte de este trabajo, dado que permite conocer la representación social que las personas poseen de rol y aspiraciones personales vinculadas al grado en el que se encuentra respecto de los proyectos que orientan su sentido.

Podría enriquecerse la investigación con la inclusión de investigaciones alusivas al tema en otros países a fin de poder comparar la situación entre Latinoamérica con la de otros continentes. También podría realizarse una investigación empírica en aspirantes a diferentes organizaciones de seguridad.

Se sugiere en futuros trabajos de investigación ampliar la muestra y poder comparar datos utilizando el cuestionario CARAP y el PIL test al inicio de la formación y al graduarse. Del mismo modo se prevee continuar indagando en relación con las apreciaciones e ideas en relación al padecimiento que poseen personas que forman parte de una organización de seguridad en líneas de investigación futuras. Es el resumen de la investigación y su estado actual. Aquí se pueden plantear objetivos futuros a seguir en la linea de investigación. 


\section{Referencias}

Aritzeta, A. y Ayestarán S. (2003). Aplicabilidad de la teoría de los roles de equipo de Belbin: Un estudio longitudinal comparativo con equipos de trabajo. Revista de Psicología General y Aplicada 56 (1), 61-75.

Bergman, M. \& Flom, H. (2012). Determinantes de la confianza en la polícia: una comparación entre Argentina y México. Perfiles latinoamericanos, 20 (40), 97-122. Recuperado en 25 de julio de 2017, de http:/ / www.scielo.org.mx/scielo.php?script=sci_arttext\&pid=S0188-

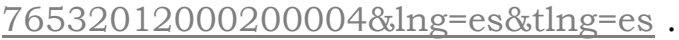

Clavijo, A; Trincheri, M. y Trincheri, D. (2014). Seguridad y Derechos humanos: Un estudio de las representaciones de policias de la Provincia de Buenos Aires. Trabajo presentado en IX Jornadas de investigación, docencia, extensión y ejercicio profesional: "Transformaciones sociales, politicas públicas y conflictos emergentes en la sociedad argentina contemporanea, La Plata.

Díaz de Dragotta, M. (2015). Aplicaciones del test persona con arma: habilitación de licencias de conducir, portación y tenencia de armas Evaluaciones psi colaborales, (1a ed.). Guaymallén, Mendoza: El autor.

Fassio A. Pascual L. \& Suarez F. (2004). Introducción a la Metodología de la Investigación Aplicada. Buenos Aires: Ediciones Macchi.

Frankl, V. E. (1994). Psicoterapia y Humanismo ¿tiene sentido la vida? México. Fondo de Cultura Económica.

Goffman, E. (1971). La presentación de la persona en la vida cotidiana. Buenos Aires. Amorrortu.

Gottfried, A. E. (2016). Adaptación Argentina del PIL Test (Test de Sentido en la Vida) de Crumbaugh y Maholick. Revista de Psicología, 12 (23) recuperado el 3 de mayo del 2019 de http:/ / bibliotecadigital.uca.edu.ar/greenstone/cgibin/library.cgi?a=d\&c=Revistas\&d=adapta cion-argentina-pil-test.pdf

Gottfried, A. E.(2017). Manual del Test de Sentido en la vida. Evaluación Cuantitativa e interpretación Cualitativa del PIL Test (Purpose in Life Test) de Crumbaugh y Maholick. Mendoza. GLE.

Hernández Sampieri, R; Fernández Collado, C y Baptista Lucio, P. (2010). Metodología de la investigación (6a ed.). México D.F, Mc Graw Hill Education.

Klos, M. C y Gutiérrez De Vázquez, M. (2016). Motivación vocacional-ocupacional, valores y estilos de liderazgo en cadetes del ámbito policial. Revista acta psiquiátrica y psicológica de América latina 62 (4) 231-240.

Lanosa, H. (2016). Valores para una Vida espiritual. Buenos Aires.Pymedia S.A.

Losada, A. V. (2014). Uso en Investigación y Psicoterapia del Consentimiento Informado. En Kerman, B y Rodríguez Ceberio, M. E. En búsqueda de las ciencias de la mente. Buenos Aires: Editorial Universidad de Flores.

Losada, A. V. (2017). Sociopsiconeuroinmunoendocrinología. Contribución teórica de la relación entre la sociología y la psiconeuroinmunoendocrinología. Revista Mexicana de Investigación en Psicología, 9(1), 73-80.

Losada, A. V. (2019). Interdisciplinariedad y Sociopsiconeuroinmunoendocrinologias. Revista Neuronum, 5(1), 154-166. 
Losada, A. V, Rivela, C. V \& Otero, V. (2019). Consideraciones acerca del "Cuestionario de Apreciación sobre Rol y Aspiraciones personales" en personas que forman parte de una organización de seguridad. Revista Observatorio Digital 5 (1) 497-527.

Martinez, E (2009). Buscando el Sentido de Vida. Manual del Facilitador. Bogotá. Colectivo Aquí y Ahora.

Montero, I. y Leon, O.G. (2007). A guide for naming research studies in Psychology. International Journal of clinical and health Psychology, 7, 847-862.

Ovejero Bernal, A. (2007). Las relaciones humanas. Psicología Social teórica y aplicada. Madrid Biblioteca Nueva.

Peiró, J. M. (1990). Organizaciones nuevas perspectivas psicosociológicas. Barcelona: Leuka.

Porras Velasquez N, R. (2012). La realidad organizacional: Desde la perspectiva psicosocial Fundación Universitaria los Librertadores. Revista Iberoamericana de Psicología: Ciencia y Tecnología 5 (1); 7-18.

Rodríguez Cueto, I. (2008). El modelo de trabajo en equipo. Revista Española de Drogadependencias 33 (4) 241-245.

Rodríguez Salazar, T. y García Curiel, M. (2007). Representaciones Sociales, teoría e investigación Universidad de Guadalajara centro universitario de Ciencias Sociales y Humanidades. México: CUCSH-UDG.

Schvarstein, L (1991). Psicología Social de las Organizaciones: Nuevos Aportes. Buenos Aires. Paidos.

Sirimarco, M. (2009). El abordaje del campo policial. Algunas consideraciones en torno a la formación inicial: entre la praxis y las reformas. Revistas Jurídicas, 6 (2) 123 - 139. 